\title{
The mediational role of intimate partner acceptance and psychological adjustment in the relationship between intimate partner control and marital adjustment
}

\author{
Ayşegül Aracı liyiaydın ${ }^{1}$ (D) $\cdot$ Zeynep Hatipoğlu Sümer ${ }^{2}$
}

Accepted: 3 February 2021 / Published online: 6 March 2021

(C) The Author(s) 2021

\begin{abstract}
Grounded in Interpersonal Acceptance-Rejection Theory (IPARTheory), this exploratory study investigated the associations among perceived intimate partner rejection, intimate partner control, psychological maladjustment, and marital adjustment. Perceived behavioral control in intimate partner relationships has been under-researched within the context of IPARTheory. Although the role of behavioral control in child/adolescent-parent relationships has been well-established, insufficient exploration of the phenomenon in marriage relationships calls for new empirical findings. The conveniently selected sample consisted of 624 (360 female, 264 male) married individuals living in big cities of Turkey. Intimate Partner Acceptance-Rejection/Control Questionnaire, Personality Assessment Questionnaire, Dyadic Adjustment Scale, and Demographic Form were utilized to gather data. Structural Equation Modeling was utilized as the primary analysis to test the proposed sequential mediational model of the study. The results indicated that intimate partner behavioral control was significantly and indirectly associated with marital adjustment through the mediating effects of intimate partner rejection and psychological maladjustment. Moreover, intimate partner rejection had direct effects on psychological maladjustment and marital adjustment. The indirect effect of intimate partner rejection on marital adjustment via the mediation of psychological maladjustment was found to be significant. Lastly, the sequential mediation by intimate partner rejection and psychological maladjustment in the relationship between intimate partner control and marital adjustment was also significant. This study adds to the existing literature on IPARTheory by showing that perceived intimate partner behavioral control is quite negatively related to marital adjustment. Results underscore how perceived behavioral control by a spouse triggers rejection and diminishes the psychological adjustment of the controlled partner.
\end{abstract}

Keywords Interpersonal acceptance and rejection theory $\cdot$ Intimate partner control · Psychological adjustment · Marital adjustment $\cdot$ Intimate partner acceptance

\section{Background}

Marital adjustment is one of the widely studied constructs in marriage literature as a dimension of marital interaction or as an indicator of marriage functioning. So far, many studies have sought to explain and explore the ingredients of a healthy,

Ayșegül Aracı İyiaydın

aysegul.iyiaydin@tedu.edu.tr

Zeynep Hatipoğlu Sümer

zeynep@metu.edu.tr

1 TED University, Ziya Gökalp Street No: 48, 06420, Çankaya Ankara, Turkey

2 Middle East Technical University, Dumlupınar Street No:1, 06800, Çankaya Ankara, Turkey good-functioning marriage by using numerous theories and assumptions. A systematic study of marital adjustment continues to evolve due to the social significance of revealing how and why marriages vary in their quality considering the complex range of factors (Bradbury, Fincham, \& Beach, 2004). Among several individual, relational, and environmental factors shown as predictors of marital adjustment, the Parental Acceptance-Rejection Theory (PARTheory) or Interpersonal Acceptance-Rejection/Control Theory (IPARTheory) as the most up-to-date version of the name, has provided a framework to study intimate relationships. IPARTheory is an evidencebased theory of socialization and lifespan development that intends to explore and predicts major causes, consequences, and other correlates of acceptance-rejection and control in intimate relationships cross-culturally (Rohner, 2016). Acceptance refers to the warmth, or simply love that a person perceives from his/her intimate partner and which is expressed through 
physical, verbal, or by means of purely symbolic but culturally constructed indicators of support or approval, whereas rejection corresponds to the perceived absence or significant withdrawal of warmth, affection, care, support, and concern of an individual from his/her intimate partner (Rohner, 2016). Perceived intimate partner control is about the extent to which one places limits or restrictions on his/her intimate partner's behaviors (Rohner, 2005).

Rohner (2016), the developer of the IPARTheory, asserts that humans strongly in need of acceptance from parents and other attachment figures no matter what culture, gender, age, ethnicity, or other such determinant conditions are. When this need is not satisfied properly, they worldwide tend to disclose themselves to be hostile and aggressive, dependent or defensively independent, impaired in self-esteem and self-adequacy, emotionally unstable, and to have a negative worldview, which are significant indicators of psychological maladjustment. The form, frequency, severity, and duration of perceived rejection determine the extent of maladjustment though. Not only acceptance-rejection, but also the control dimension of the IPARTheory is related to specific consequences on the psychological adjustment of children and adolescents, yet the role of control in intimate partner relationships has not sufficiently been studied. Theoretically, it was assumed that perceived behavioral control by an intimate partner would be associated with psychological adjustment and relationship functioning negatively, and probably through its association with perceived rejection.

Controlling behaviors towards one's intimate partner have been regarded as a type of psychological abuse encompassing behaviors like ridiculing, verbal threatening, isolating from family and friends, and controlling of one's partner to degrade and attack self-worth of her/him (Lawrence, Yoon, Langer, \& Eunyoe, 2009). Concerning that definition, perceived intimate partner behavioral control could be evaluated as a form of relationship violence and abuse. In that respect and when taken as an abusive act, researchers have long acknowledged the link between intimate partner abuse of any kind and numerous variables like low self-esteem, depression, anxiety, low marital satisfaction, and low marital adjustment on negative psychological outcomes and marital quality. For instance, a body of research showed the precursor role of controlling behaviors from an intimate partner on the likelihood of physical and sexual violence towards the partner or the spouse (e.g., Antai, 2011; Graham-Kevan \& Archer, 2008; Hunt, 2007), which was directly and negatively affecting dyadic adjustment or satisfaction of couples. Notwithstanding the fact that perceived intimate partner control has not yet been addressed as a type of relationship violence in IPARTheory, the literature says so as being a type of psychological aggression to control the partner's behaviors and preferences. Rejection concept in IPARTheory is characterized by indifference/neglect, hostility/aggression, and lack of warmth/affection towards the spouse, all of which are also treated as components of relationship violence as well all of which can also be somehow regarded as components of relationship violence. Meanwhile, a behaviorally controlled partner may feel rejected due to not being respected for his/her preferences and needs, which in turn diminishes his or her marital adjustment level.

In current literature encompassing the past 20 years, the concept of marital adjustment has been explored with a great variety of psychological constructs as being related to psychological adjustment or maladjustment. In the present study, the psychological adjustment was addressed in the framework of the Interpersonal Acceptance-Rejection and Control Theory. The bidirectional association between psychological adjustment and marital adjustment has consistently been demonstrated in the literature (e.g., Epözdemir, 2012; Kamp-Dush, Taylor, \& Kroeger, 2008; Shek, 2001; Villeneuve et al., 2014; Whisman \& Bruce, 1999; Yeşiltepe \& Çelik, 2014). In other words, marital adjustment influences and is influenced by psychological adjustment. As we proposed in our hypothesized model in Fig. 1, the link from the psychological adjustment to marital adjustment would have some underlying implications. There is much empirical evidence showing the strong association between parental and/or intimate partner acceptance/rejection and psychological adjustment of the individual (Chyung \& Lee, 2008; Khaleque \& Rohner, 2012; Varan, Rohner, \& Eryüksel, 2008). Individuals who experienced intimate partner rejection are supposed to believe that they are unworthy of love and because of impaired self-esteem and misinterpretation of the actions of others in a negative way, their satisfaction in their close relationships decreases (Rohner, 2004). On the contrary, perceived intimate partner acceptance is associated with a better psychological adjustment encompassing higher self-esteem and self-adequacy, emotional responsiveness, positive worldview, lower aggression/hostility, higher emotional stability, and healthy independence. Those elements in one's psychological adjustment would contribute to regulating his/her emotions, investing in relationships based on emotional and physical availability, trusting oneself and others, maintaining hope, resolving conflicts, managing and compromising negative situations, and sustaining intimate bonds; all of which could be necessary ingredients of a close and satisfying marital relationship or better adjustment in the marriage. To sum up, the extent to which one perceives acceptance or rejection and control from his/her husband or wife would empower or deteriorate the marital concord between couples.

A meta-analysis of 93 cross-sectional and longitudinal studies (Proulx, Helms, \& Buehler, 2007) on the association between marital quality and personal well-being demonstrated that married individuals having a better quality of marriage relationship reported less depressive symptomatology, more self-esteem, life satisfaction, global happiness, and physical health ( $r$ was .37 for cross-sectional and .25 for longitudinal 
Fig. 1 The hypothesized model with standardized estimates and significant and nonsignificant paths. Only the latent variables were included in the figure. Solid lines indicate significant and dashed lines indicate nonsignificant path

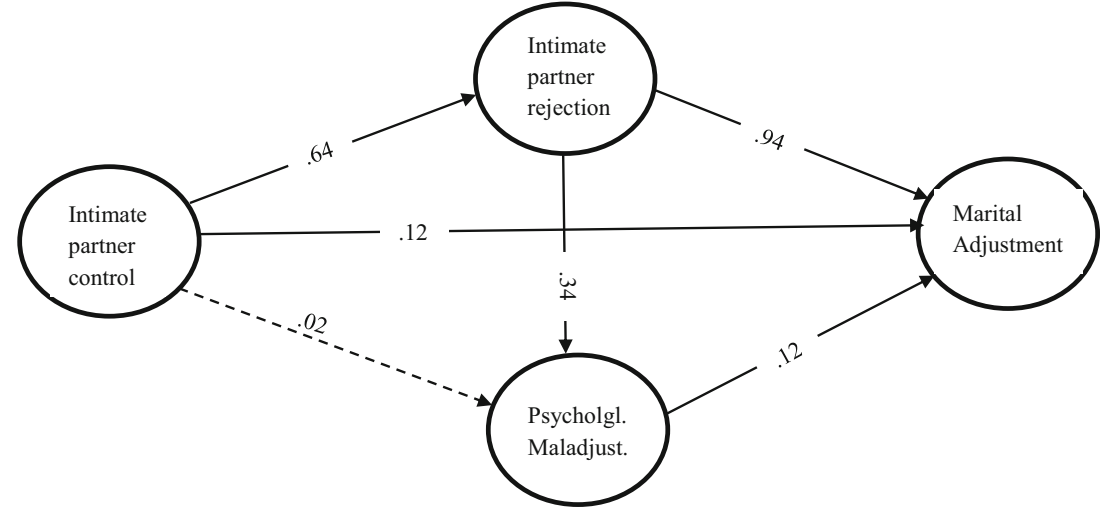

effects). Moreover, marital discord and satisfaction were found significantly and negatively related to intimate partner violence $(r=-0.27)$ in another meta-analysis of 32 articles (Stith, Green, Smith, \& Ward, 2007). It is likely to deduce from these results that good functioning marriage buffers individuals against well-established risk factors of well-being. Heretofore, controlling behaviors in the marriage relationship based on the IPARTheory framework has been underresearched, contrary to its prevalent inspection in the parentchild relationship. Thereby, the role of perceived intimate partner control needs to be clarified by offering novel and reasonable models to ascertain possible direct associations and underlying mechanisms operating between perceived behavioral control and marital adjustment of couples. In line with the theoretical knowledge and research findings based on the conceptual framework of Interpersonal AcceptanceRejection/Control Theory (IPARTheory), we investigated the mediator roles of intimate partner rejection and psychological maladjustment in the presumed relationship between intimate partner control, and marital adjustment. We hypothesized that perceived intimate partner control will be negatively associated with marital adjustment through mediating effects of perceived intimate partner rejection and psychological maladjustment as depicted in Fig. 1. Besides, there will be several direct relationships among intimate partner acceptance, psychological adjustment, and marital adjustment. Moreover, psychological maladjustment will mediate the link between intimate partner rejection and marital adjustment.

\section{Method}

\section{Participants}

Data were collected from 624 married individuals living in different urban areas of Turkey. Of the 624 participants, 264 were male $(42.3 \%)$ and 360 were female $(57.7 \%)$. The ages of the participants ranged between 20 and 63 with a mean age of $36.82(\mathrm{SD}=8.19)$. Average marriage year of the participants was 9.29 (minimum $=1 ;$ maximum $=33 ; \mathrm{SD}=7.99$ ). The majority of the participants were at least have an undergraduate degree $(70.4 \%)$ and more $(29.6 \%)$; that is to say, most of the participants were from a quite high educational background profile. In terms of the number of children they have, 189 of the participants $(30.3 \%)$ had no children, 239 of the participants (38.3\%) had one child, 167 of the participants (26.8\%) had two children, and 29 of the participants had three or more children (4.6\%). All of the participants were in their first marriages.

\section{Data Collection Instruments}

Demographic Information Form The researchers developed a demographic form in which participants were asked to specify their gender, age, length of the marriage, educational background, and the number of children.

Intimate Partner Acceptance-Rejection and Control Questionnaire (IPAR/CQ) The Intimate Partner AcceptanceRejection/Control Questionnaire was developed by Rohner (2001) to measure one's perceived acceptance-rejection and behavioral control experienced in the relationship with his/her intimate partner. The acceptance-rejection part of the questionnaire consists of 60 items, comprised of four subscales as warmth/affection, indifference/neglect, hostility/aggression, and undifferentiated rejection. Respondents were requested to report their level of agreement on a Likert-type scale ranged from (1) almost never true to (4) almost always true, and the overall score varied between 60 and 240, with higher scores indicating greater rejection from an intimate partner and lower scores referring to greater acceptance perceived. The behavioral control part of the questionnaire includes 13 items such as "My partner tells me how to behave" and "My partner wants to know my whereabouts". Scores 
obtained from this part ranged from a minimum of 13 (referring maximum to permissive control) to a maximum of 52 (representing extreme restrictive control).

Rohner (2005) showed that IPAR/CQ is a valid and reliable instrument with the internal consistency reliability coefficients as .74 for the acceptance-rejection part and .85 for the behavioral control part for the American sample. Test-retest reliability of the entire IPAR/CQ was found as .97 over the span of 4 weeks. In factor analysis, acceptance and rejection emerged as two independent factors in the first part. Regarding the control portion, permissive and strict factors were reported to emerge.

Turkish adaptation of the IPAR/CQ was conducted by Varan (2003) with 1700 participants from clinical and nonclinical samples between the ages of 17 and 78 years. The Cronbach alpha value for the overall questionnaire was found as .97, for the first four subscales were ranged between .85 and .96. The construct validity of the questionnaire was explored via factor analysis and it was concluded that rejection and acceptance factors explained $72.26 \%$ of the total variance.

The Cronbach alpha coefficient for the Control subscale was calculated as .88 by Varan (2003). To explore the construct validity of the Control subscale, factor analysis was performed. "Permissiveness" and "strictness" as emerging two factors explained $82 \%$ of the variance of the Control subscale. Consequently, Varan (2003) stated that the IPAR/CQ was a valid and reliable questionnaire for the Turkish sample. In the current study, Cronbach's alpha as the reliability index of the questionnaire was found .84 for the total scale, .74 for the acceptance-rejection subscale, and .88 for the behavioral control subscale.

Adult Personality Assessment Questionnaire (PAQ) The Adult PAQ (Rohner, 2005) is a self-report questionnaire designed to assess individuals' perceptions of themselves to seven personality dispositions: (a) aggression/hostility, (b) dependency, (c) self-esteem, (d) self-adequacy, (e) emotional responsiveness, (f) emotional stability, and (g) worldview. The questionnaire has 63 items total, 9 items in each seven subscales with a 4point Likert-type response scale from 4 (almost always true) to 1 (almost never true). The scales are designed to measure the personality characteristics described in IPARTheory's personality theory. By summing the scores of seven scales, a profile of self-reported psychological adjustment of the respondent is obtained. This is the form of adjustment or maladjustment predicted in IPARTheory to be universally associated with the experience of acceptance-rejection by attachment figures (parents, intimate partners, etc.) throughout life. The PAQ ranges from a possible low score of 63 to a possible high score of 252. A higher score refers to higher psychological maladjustment reported by the individual. The items are written in the present tense and ask respondents their actual feelings about themselves-rather than ideal or desired ones (Rohner \& Ali, 2016).
Psychometric properties of the Adult Psychological Adjustment Questionnaire were examined by Varan (2003) in Turkish culture with $1700 \mathrm{clinical} /$ non-clinical participants aged from 17 to 78 years. The author reported in the unpublished study that total internal consistency Cronbach alpha was found as .91. For the subscales, alpha levels changed between .68 and .82. The construct validity of the questionnaire was searched via factor analysis, and the emerging six factors explained $71.52 \%$ of the variance in adult psychological adjustment.

In light of this evidence, Varan (2003) concluded that the Adult Personality Assessment Questionnaire (PAQ) measures the psychological adjustment levels of Turkish adults in a valid and reliable manner. In the current study, Cronbach's alpha coefficient of the questionnaire was found as .94 for the total scale.

Dyadic Adjustment Scale (DAS) The Dyadic Adjustment Scale (DAS) was developed by Spainer (1976) to measure the marital quality of couples. The scale consists of 32 items to assess dyadic satisfaction, dyadic cohesion, dyadic consensus, and affectional expression, all of which are considered as the four basic components of a relationship functioning between couples. There are 5-points, 6-points, 7-points Likert type items ranging between "always disagree" to "always agree" and dichotomous items like "yes or no" answers in the scale. The total score of the DAS changes between 0 and 151 with higher score referring to a higher perception of the marital quality of the relationship. The Cronbach alpha values of the scales were ranged from .73 to .94 for the four subscales and for the entire scale it was reported as .96 .

Turkish adaptation of the Dyadic Adjustment Scale (DAS) was carried out by Fışıloğlu and Demir (2000) with 264 married individuals who were in their first marriages (132 female, 132 male). To test the construct validity of the Turkish DAS, a principal component analysis was used to confirm the dimensions (dyadic satisfaction, dyadic cohesion, dyadic consensus, and affectional expression) as aspects of marital adjustment. The four factors explained $45.5 \%$ of the total variance in marital adjustment. The total internal consistency reliability score of the Turkish DAS was reported as .92. For the criterion validity, the correlation between Locke-Wallace Marital Adjustment Test and the Turkish DAS was $r=.82$. The mean score of the sample on the total scale was found as 103.7. The researchers stated that the Turkish DAS has sufficiently high reliability and construct validity to be used to assess marital adjustment levels of married individuals. In the present study, Cronbach's alpha coefficient of the questionnaire was calculated as .95 for the total scale.

\section{Data Collection}

Prior to the data collection process, several procedures were followed. Firstly, the protocol of the study was approved by 
the Middle East Technical University Human Subjects Ethics Committee. Secondly, authors of the instruments were contacted via e-mail, and their permissions to use the questionnaires were obtained. And then, the authorities in the institutions whereby the participants being recruited were visited to introduce the study and take consent for getting the emails of potential participants. With other participants in the personal network of the researcher, email addresses and the WhatsApp application were used to deliver the survey. The rationale behind using the WhatsApp application was its' practicality for a potential participant to reach the measures of the study via clicking on the link.

Later, soft versions of the instruments along with an informed consent form were created in an online platform named Google Forms which is a website permitting to generate online surveys. An online link was formed through which participants were directed to the surveys of the study. Only married individuals, who were married at least for a year and dwelling with their husbands or wives, were invited to participate. Confidentiality and anonymity were ensured by adjusting the settings of the survey program and informing the participants in the introduction paragraph of the online survey protocol. In order to prevent couple interaction while filling out the survey, it was requested and emphasized that they be alone.

\section{Preliminary Analyses}

In the first step, we used descriptive statistics to describe the participants of the study. Differences regarding gender in terms of study variables were analyzed with independent samples t-tests by using SPSS 21 statistical package program (IBM Corp, 2012) to decide on testing the hypothesized model of the study for the whole sample or separately for women and men. Bivariate correlations among study variables were also computed. Along with the aim of the study, which was to explore the associations among perceived intimate partner rejection, perceived intimate partner behavioral control, psychological maladjustment, and marital adjustment, we employed Structural Equation Modeling analysis with latent variables, using AMOS 21 software (Arbuckle, 2014).

Prior to the main analyses, we first checked the assumptions necessary to conduct SEM analysis (missing data, sample size, outlier analysis, multivariate normality, linearity and homoscedasticity, and multicollinearity), and we found no violation in each of them. Means and standard deviations of the study variables for the total sample along with bivariate correlations are presented in Table 1. To test possible gender differences on study variables, we run several independent samples t-tests. Before conducting the independent samples t-test analyses, homogeneity of variances between groups was assessed by Levene's tests. Except for the marital adjustment variable, the results of the tests produced a non- significant $p$ value $(p>.05)$, which was enough to conclude that homogeneity of variances was ensured between the groups. If homogeneity of the variances is violated, SPSS produces an alternative $t$ value. For the marital adjustment variable, $t$ value in case of equal variances not assumed was used. Alpha level was set to .001. Before running the tests for comparisons and to reduce the Type I error which was a possibility due to multiple comparisons, Bonferonni correction was applied by adjusting $\mathrm{p}$ value to $.0125(.05 / 4)$. According to the results of independent tests for multiple comparisons, there were no significant differences between scores of male and female participants on intimate partner rejection $[t$ $(624)=.54, p=.58]$, psychological maladjustment $[t(624)=$ $-1.01, p=.31]$, and marital adjustment $[t(624)=1.12$, $p=.26]$. But men reported significantly higher partner control than women $[t(624)=7.18, p=.000]$. When taken into account the cutoff scores of the Intimate Partner Control Questionnaire, it could be possible to conclude that both men and women perceived moderate control from their intimate partners on average in the current study. Therefore, we did not perform model testing separately for men and women.

For the interpretation of the results of SEM analysis, we used several criteria including model fit indices, squared multiple correlations (SMC), and parameter estimates. As a criterion for evaluating the correlation and beta coefficients, we utilized Cohen (1988), which suggests considering correlations from .10 to .29 as weak, from .30 to .49 as moderate, and from .50 to 1.00 as strong.

\section{Results}

As can be seen in Table 1, marital adjustment was negatively and moderately correlated with intimate partner control $(r=$ $-.40, p<.01)$, negatively and moderately correlated with psychological maladjustment $(r=-.34, p<.01)$; and negatively and strongly correlated with intimate partner rejection $(r=$ $-.86, p<.01)$. Except for correlation results regarding the marital adjustment of being the dependent variable of the study, there were significant correlations among independent variables as well. It was found a significant and moderate to strong positive correlation between perceived intimate partner rejection and perceived intimate partner control $(r=.49$, $p<.01$ ), meaning that the more "behaviorally controlling" spouses seem to be, the less loving or accepting they perceived to be. Moreover, perceived intimate partner rejection and psychological maladjustment were positively and weakly correlated $(r=.29, p<.01)$. And lastly, perceived intimate partner control was positively and weakly correlated with psychological maladjustment $(r=.18, p<.01)$.

Afterward, we computed partial correlations among intimate partner control, psychological maladjustment, and marital adjustment after controlling the effect of intimate partner 
Table 1 Intercorrelations, means, and standard deviations for the study variables

\begin{tabular}{|c|c|c|c|c|c|c|c|c|}
\hline \multirow[t]{2}{*}{ Variables } & \multirow[t]{2}{*}{1} & \multirow[t]{2}{*}{2} & \multirow[t]{2}{*}{3} & \multirow[t]{2}{*}{4} & \multirow[t]{2}{*}{ Possible range } & \multirow[t]{2}{*}{ Actual range } & \multicolumn{2}{|c|}{ Total $(N=624)$} \\
\hline & & & & & & & M & $\mathrm{SD}$ \\
\hline 1. Intimate Partner Control & - & $-.49 * *$ & $-.18 * *$ & $-.40 * *$ & $13-52$ & $13-52$ & 28.03 & 8.21 \\
\hline 2. Intimate Partner Acceptance & & - & $.29 * *$ & $.86^{* *}$ & $60-240$ & $60-237$ & 93.88 & 31.84 \\
\hline 3. Psychological Adjustment & & & - & $.34 * *$ & $63-252$ & $69-210$ & 121.09 & 25.31 \\
\hline 4. Marital Adjusment & & & & - & $0-151$ & $20-150$ & 111.98 & 22.63 \\
\hline
\end{tabular}

rejection for both males and females. The correlation between intimate partner control and psychological maladjustment $(r=.18, p<.01)$ along with the correlation between intimate partner control and marital adjustment $(r=-.40, p<.01)$ disappeared. Then, it was noticed that the role of intimate partner control on psychological maladjustment and marital adjustment had stemmed from its association with intimate partner rejection, it was just an artifact of the correlation with perceived rejection. Based on this finding, the proposed model of the study was grounded by embedding the intimate partner rejection as a mediator variable.

Results for the hypothesized model revealed an acceptable fit to the data of the study. Chi-square value was non-significant $\chi 2$ $(2)=2466,177(p>.05)$. The normed chi-square value was $\chi 2$ / $\mathrm{df}=7.169 ;$ RMSEA $=.096($ CI $90 \%=.93-1.00) ; \mathrm{CFI}=.833$; $\mathrm{TLI}=.816$; and SRMR $=.0766$. Model $\chi 2$ is sensitive to sample size, and Chi-square statistic nearly always rejects the model if larger sample sizes are used (Bentler \& Bonnet, 1980). The value of RMSEA is within the acceptable range and regarded as a mediocre fit (MacCallum, Browne, Sugawara, \& M., 1996). The value of SRMR is deemed acceptable, but CFI is mediocre (Hu \& Bentler, 1999). The model fit indices could be negatively affected by (1) the relatively large sample size of the current study, (2) a strong correlation between intimate partner rejection and marital adjustment $(r=-.84)$, and (3) the high number of parameters in the proposed model. As Thompson (1999), a general rule of thumb may not be always achievable, and model fit remains a central question in SEM analysis. All the factor loadings were significant and ranged between .23 and .95 .

To explore the amount of variance explained by the proposed serial mediational model, we checked the squared multiple correlations $\left(\mathrm{R}^{2}\right)$. The results showed that intimate partner control explained $41 \%$ of the variance in intimate partner rejection. Both intimate partner control and intimate partner rejection accounted for $13 \%$ of the variance in psychological maladjustment. Besides, the overall model explained $85 \%$ of the variance in marital adjustment.

The bootstrapped results of direct, indirect, and total estimates with bias corrected (BS) percentile intervals with 95\% confidence are reported in Fig. 1. According to the bootstrapped results, intimate partner control had a nonsignificant direct effect on psychological maladjustment $(\beta=.02, S E=.06, p>.05)$, but significant on marital adjustment $(\beta=-.12, S E=.03, p<.01)$. The direct effects of intimate partner rejection $(\beta=-.94, S E=.03, p<.01)$ and psychological maladjustment $(\beta=-.12, S E=.03, p<.01)$ on marital adjustment were also significant, former strong latter weak in effect. Intimate partner rejection had a significant direct effect on psychological maladjustment with a moderate effect size $(\beta=.34, S E=.06, p<.01)$. The direct effect of psychological maladjustment on marital adjustment was also significant $(\beta=-.12, S E=.03, p<.01)$ with a weak effect size. Therefore, participants who perceived more rejection from their spouses and reported worse psychological adjustment depicted lesser marital adjustment of their own. Likewise, the ones who had a low perceived behavior control coming from their intimate partner perceived less rejection in their marriage relationship as well. Lastly, as rejection perceived by the spouse increased, the psychological adjustment of the participant also got worse. Perceived intimate partner control did independently contribute to psychological maladjustment and marital adjustment, yet to a few extents.

We explored four indirect effects in the hypothesized model, all of which were significant. The first one, which was the indirect effect of control on psychological maladjustment via intimate partner rejection was significant $(\beta=.22, S E=.04$, $p<.01,95 \%$ CI $[.150, .304])$ with a weak to moderate effect size. For the indirect effect of intimate partner control on marital adjustment via intimate partner rejection, the result was significant $(\beta=.22, S E=.04, p<.01,95 \%$ CI $[.150, .304])$ and weak to moderate in effect. Regarding the indirect effect of intimate partner rejection on marital adjustment via psychological maladjustment $(\beta=-.04, S E=.01, p<.01,95 \%$ CI $[.022, .067])$, it was significant and weak in effect. The last inspected and significant indirect effect $(\beta=-.03, S E=.00$, $p<.01,95 \%$ CI $[-.023,-.006])$ was intimate partner control on marital adjustment via sequential intimate partner rejection and psychological maladjustment, respectively with a weak effect size. In sum, participants who perceived high control from their spouses reported higher psychological maladjustment and lower marital adjustment via a higher perception of rejection in their relationships. Those who perceived their partners as more rejecting had a lower marital adjustment via an increase in their psychological maladjustment. 
Furthermore, participants who perceived higher control from their spouses reported lesser marital adjustment through an increase in their rejection perception and psychological maladjustment sequentially.

In terms of total effects, we found that intimate partner $\operatorname{control}(\beta=-.52, S E=.04, p<.01)$ had significant total effect on marital adjustment with a strong effect size. The addition of intimate partner rejection and psychological maladjustment increased the $\beta$ value of intimate partner control on marital adjustment from -.12 to -.52 . Similarly, intimate partner rejection had a significant total effect on marital adjustment $(\beta=.98, S E=.03, p<.001)$ with a quite strong effect size. To sum up, results on both direct, indirect, and total effects showed that the most powerful predictor in marital adjustment is the degree of rejection one perceives from his/her spouse in the marriage relationship.

\section{Discussion}

In the current study, we obtained significant findings on the mediating role of perceived intimate partner rejection and psychological maladjustment in the relationship between perceived intimate partner control and marital adjustment. What is novel in this study was the attempt to generate a model, in which perceived intimate partner rejection, as well as psychological maladjustment, are the agents for perceived intimate partner behavioral control to transfer its impact on marital adjustment. Indeed, this mechanism was assured, yet not with the robust effect size for the serial mediation path. However, the mediating effect of intimate partner rejection in the association between intimate partner control and marital adjustment as well as psychological maladjustment was quite illuminating. Studies aimed to search the dynamic of perceived intimate partner behavioral control on psychological maladjustment and relationship outcomes have not gone beyond roughly assuming the link (mostly descriptive in nature) between behavioral control and acceptance-rejection without testing it thoroughly. Hence, this study could be evaluated as a first systematic effort to include and explore the behavioral control part of the IPARTheory in the context of a marital relationship by using an inferential statistics method rather than reporting only the descriptive part. Furthermore, we proposed and found evidence on the mechanism through which perceived behavioral control operates, so building upon the existing accumulation of empirical knowledge on the issue.

The surprising finding of this study was the outstanding explanatory power of perceived intimate partner rejection on marital adjustment. Most of the variance accounted for $75 \%$ of marital adjustment resulted from perceived intimate partner rejection. This result made us think again and again with suspicion on the likelihood of having measured the same thing with intimate partner rejection and marital adjustment questionnaires. However, there were sound reasons to eliminate this option. First, the literature pertaining to both variables has treated them as separate constructs. The first one is dealing with the warmth/affection part of a marriage relationship whereas the latter is designed to measure aspects regarding satisfaction, consensus, affectional expression, and cohesion. Secondly, bivariate correlations (especially between warmth-affection subscale of the IPAR/Q and affectional expression subscale of the DAS) among the subscales of the two questionnaires were cautiously examined one by one, and no multicollinearity (the criterion of Tabachnick \& Fidell, 2014 was used as $r \geq .90$ ) was detected (correlation coefficients were changed between .54 and .78) in any of all binary combinations. Eventually, we concluded that perceived intimate partner rejection is a critical component of marital adjustment. In other words, the marital adjustment level of a spouse seems to be highly associated with the extent or degree of rejection being perceived from husband or wife.

So far, the attempts to design studies based on prediction have been quite limited to the association between intimate partner rejection and marital adjustment. Nevertheless, Rohner (2008) explained the warmth dimension of intimate partner rejection as encompassing lack of affectional bond, love, nurturance, comfort, trust, support, empathy, self-verification, respect, shared responsibility, sensitivity, equality, and the behaviors (physical, verbal, and symbolic) being showed to communicate or express these feelings to the partner. In this respect, the marriage literature is quite prosperous that documented robustly the positive impact of these factors on marital quality related constructs like marital adjustment, marital satisfaction, and marital happiness (e.g., Allen \& Thompson, 1984; Çağ \& Yıldırım, 2013; İnal, 2014; Mcdonald, Olson, Lanning, Goddard, \& Marshall, 2018; Öztürk, 2017; Sacco \& Phares, 2001; Weger, 2005; Wilcox \& Nock, 2006). Then, it could be speculated that such a strong association would be an outcome of the construct per se, that is rejection being connected with or harboring a lot of factors in itself as mentioned above. It would not be too wrong to note that rejection can be evaluated as an umbrella term encompassing many feelings that are proved to be benchmarks of a good-functioning marriage relationship. Besides, the need to feel accepted by significant others is a basic human need that has been strongly emphasized by many theorists to date (e.g., Bowlby, 1969/ 1982; Rohner, 1986).

Additionally, the conjugal context has apparently the potential to represent the most significant other for many of us, except for the parent-child context. Prominent in this regard is another but most likely facet in an array of possible explanations of the strong association between intimate partner rejection and marital adjustment: the central importance of the need to feel accepted by significant others. Feelings and related behaviors, encapsulated in intimate partner acceptance that was mentioned previously, may foster constructive dyadic 
interactions in such a way that yields couples being satisfied, reaching a consensus on matters, expressing affection, and engaging in joint activities in their marriages.

Moreover, we significantly generated a model to test the mediating role of perceived intimate partner rejection in the relationship between perceived intimate partner control and psychological maladjustment, and between perceived intimate partner control and marital adjustment. The direct positive association between perceived intimate partner control and perceived intimate partner rejection could have stemmed not only from being a sign of interference to the quest for independence and autonomy in the marriage relationship but also from the likelihood of triggering aggression on behalf of the controlling partner due to non-compliance of the controlled spouse. Previous studies (e.g., Eryavuz, 2006; Khaleque, Rohner, \& Laukkala, 2008; Rohner \& Khaleque, 2008) have shown the link between perceived intimate partner behavioral control and perceived intimate partner rejection, though most of them were limited to descriptive findings. These studies denoted that the more behaviorally controlling partners were perceived to be less loving or accepting. The starting point of these studies was the evidence that the extremes of behavioral control perceived by parents (extreme permissiveness and extreme restrictive control) tended to be associated with parental rejection, especially with parental hostility and aggression. Upon this observation, we inspected bivariate correlations among the scores of warmth/affection, hostility/aggression, indifference/neglect, and undifferentiated rejection dimensions, being the subscales of intimate partner acceptance, along with intimate partner behavioral control. Then, we compared the correlation coefficients of all binary relationships by conducting Fisher's Z-test. We surprisingly found that the correlation between intimate partner control and hostility/ aggression part of intimate partner rejection $(r=.59)$ was significantly higher than warmth/affection $(r=-.43)$, indifference/neglect $(r=.29)$, and undifferentiated rejection $(r=.52)$ subscales $(p<.01)$. Then, it could be speculated that a controlling partner may show aggression or hostility towards his/her spouse especially if the spouse does not obey or comply with the proscriptions and prescriptions imposed by the partner. It is also important to notice that the other subdimensions were also related to behavioral control. Even if the spouse did not refuse the rules and directives, s/he might think to be not respected for his/her preferences and decisions, which in turn might lead to feeling not accepted. Thus, the attempts or acts of controlling behavior of a partner could have been attributed by the spouse as being not accepted for his/her decisions, preferences, feelings, and thoughts, etc. This could in turn impinge upon the marital adjustment of the controlled partner negatively. To date, no empirical finding has attempted to explore a possible mediating variable between perceived intimate partner control and marital adjustment. Hence, this finding of the current study was discussed only with literature showing direct and binary associations, that is nonsignificant one between intimate partner control and marital adjustment (e.g., Eryavuz, 2006; Gökmen, 2011) and significant one between intimate partner rejection and marital adjustment (e.g., Eryavuz, 2006; Khaleque et al., 2008; Rohner \& Khaleque, 2008).

To date, existing yet too few studies on the behavioral control dimension of intimate partner relationships in the context of IPARTheory were unable to go beyond reporting descriptive correlations. Most of them measured the control dimension but excluded it in further analyses. The study has not only put the behavioral control dimension under the microscope but also has advanced the understanding of its role in marital adjustment via introducing a mediational process that was intimate partner rejection. As a first attempt to propose and test such a model, perceived behavioral control seems to operate through perceived intimate partner rejection on the associations with psychological maladjustment and marital adjustment. In other words, perceived behavioral control by an intimate partner was comprehended as a sign of rejection by the spouse regardless of gender, which in turn diminished the psychological and marital adjustment level of that spouse. In the parent-child and parent-adolescent relationship context, moderate or firm behavioral control by the parent, provided that it not be permissive or restrictive, has been mostly shown to be associated with positive psychological outcomes on behalf of children and adolescents. However, in the context of intimate partner relationships like marriage, the current study has revealed that perceived behavioral control of any magnitude harmed both psychological adjustment and marital adjustment of married individuals. This finding might be a worthwhile stimulus for future studies to gain a deeper understanding of the dynamics that behavioral control exerts in romantic relationships including but not limited to marriage.

In terms of research implications, obtained evidence in the current study regarding the remarkable contribution of perceived intimate partner rejection accounting for marital adjustment has been quite illuminating. Although there are a few empirical findings aimed to compare martially satisfied and not satisfied groups on the scores of perceived intimate partner rejection, they measured marital satisfaction with a single question as "Are you satisfied in your current marriage?" In the current study, the marital adjustment was measured with the Dyadic Adjustment Scale, which was justified as being a highly valid and reliable instrument designed to evaluate marital quality comprehensively $75 \%$ of marital adjustment was explained mostly by intimate partner rejection and partially by intimate partner control and psychological maladjustment. Such an amount is quite spectacular and giving a strong clue to crack the secret of a happy marriage. Indeed, it can be speculated that perceived intimate partner rejection seemed to be the crux of marital quality. What is implied in this finding is the central importance of feeling accepted by the spouse 
in a marriage relationship. Such a high variance of marital adjustment explained mostly by intimate partner rejection indicates in a way the power of encapsulation of the "acceptance" construct by itself. In other words, feeling accepted in the context of a marriage relationship symbolizes many other feelings herewith like affection, love, respect, care, support, and empathy, etc.

The current study unearthed the significance of partners' expression of behaviors communicating acceptance towards their spouses, which is characterized by feelings of love, affection, empathy, care, concern, understanding, appreciation, and validation on marital adjustment of them. Mental health practitioners working with couples especially may focus on the ways through which couples may exhibit more acceptance-related behaviors towards each other. Moreover, both for prevention and intervention purposes, skills training can be designed to foster the mutual use of behaviors (physical, verbal, and nonverbal expressions) that contribute to the feelings of acceptance in each spouse. The current study explicitly showed that perceived acceptance from an intimate partner had a tremendous impact on the marital adjustment of the spouse. Therefore, the attempts to enhance communication between couples that trigger the feelings of acceptance would be worthwhile as well as functional. Therefore, counselors would also contribute to the psychological adjustment of couples, which in this study was shown to be related to marital adjustment, too.

Furthermore, controlling behaviors towards the intimate partner were obviously interpreted as rejection, or lack of behavioral control was perceived as being accepted in the marriage relationship. Besides, behavioral control negatively and indirectly through acceptance was associated with psychological adjustment and marital adjustment of husbands and wives. Psychological counselors may use this finding to increase the awareness of husbands and wives on the counter-productive role of behavioral control in marriage.

The current study also indicated the direct role of psychological adjustment, in the case of controlling the effect of perceived intimate partner rejection and control, on marital adjustment. What is implied in this finding could be the likelihood that if a psychological counselor works through with the client, on an individual basis, to support or boost psychological adjustment of him/her, this will in effect enhance marital adjustment of the client as well. Last but not the least, less behavioral control and more acceptance-related behaviors, as well as a better psychological adjustment, would be among the counseling process goals for those practitioners who are willing to work on individuals and or couples with marital adjustment problems.

The present study had some limitations that should be taken into account while evaluating its results. Firstly, random sampling was not used in the process of participant selection and highly educated married individuals living in metropolitans participated in the study, which restricted the generalizability of its findings. Secondly, the data was based on respondents' subjective reports on questionnaires. The utility of self-report questionnaires is criticized due to some problems arising by its very nature such as social desirability. Furthermore, although participants were instructed to fill out the online questionnaires alone, couple interaction could not have been prevented if any. Lastly, as Spainer and Cole (1976) discussed, the marital adjustment could be studied best with a longitudinal design due to its being a process or a continuum. What was done in this research is to take a snapshot of the continuum from well-adjusted to maladjusted at one point in time. No matter how robust the correlation between intimate partner acceptance and marital adjustment, however, it does not speak to the issue of causation. A more promising means of addressing potential causal relations would be to examine this association with longitudinal studies. Not for causality, but for more powerful predictions, the model proposed in the current study is recommended to be retested with latent variables by using statistical procedures of structural equation modeling. Besides, the perception of intimate partner control should be studied with different group samples.

\section{Declarations}

Conflict of Interest On behalf of all authors, the corresponding author states that there is no conflict of interest.

The datasets generated during and/or analyzed during the current study are available from the corresponding author on reasonable request.

Open Access This article is licensed under a Creative Commons Attribution 4.0 International License, which permits use, sharing, adaptation, distribution and reproduction in any medium or format, as long as you give appropriate credit to the original author(s) and the source, provide a link to the Creative Commons licence, and indicate if changes were made. The images or other third party material in this article are included in the article's Creative Commons licence, unless indicated otherwise in a credit line to the material. If material is not included in the article's Creative Commons licence and your intended use is not permitted by statutory regulation or exceeds the permitted use, you will need to obtain permission directly from the copyright holder. To view a copy of this licence, visit http://creativecommons.org/licenses/by/4.0/.

\section{References}

Allen, A., \& Thompson, T. (1984). Agreement, understanding, realization, and feeling understood as predictors of communicative satisfaction in marital dyads. Journal of Marriage and the Family, 46(4), 915-921.

Antai, D. (2011). Controlling behavior, power relations within intimate relationships and intimate partner physical and sexual violence against women in Nigeria. BMC Public Health, 11, 511-522.

Arbuckle, J. L. (2014). Amos (version 23.0) [computer program]. Chicago: IBM SPSS.

Bentler, P. M., \& Bonnet, D. C. (1980). Significance tests and goodness of fit in the analysis of covariance structures. Psychological Bulletin, $88(3), 588-606$. 
Bowlby, J. (1969/1982). Attachment and loss. New York: Basic Books. Bradbury, T. N., Fincham, F. D., \& Beach, S. R. H. (2004). Research on the nature and determinants of marital satisfaction: A decade in review. Journal of Marriage and the Family, 62, 964-980.

Çağ, P., \& Yıldırım, İ. (2013). Relational and personal predictors of marital satisfaction. The Turkish Psychological Counseling and Guidance Journal, 4(39), 13-23.

Chyung, Y. J., \& Lee, J. (2008). Intimate partner acceptance, parental acceptance in childhood, and psychological adjustment among Korean college students in ongoing intimate relationships. CrossCultural Research, 42(1), 77-86. https://doi.org/10.1177/ 1069397107309857.

Cohen, J. (1988). Statistical power analysis for the behavioral sciences (2nd ed.). Hillsdale, NJ: Lawrence Earlbaum Associates.

Epözdemir, H. (2012). The effect of alexithymic characteristics of married couples on their marital adjustment. Journal of Family Psychotherapy, 23, 116-130. https://doi.org/10.1080/08975353. 2012.679902.

Eryavuz, A. (2006). Çocuklukta algllanan ebeveyn kabul veya reddinin yetişkinlik dönemi yakın ilişkileri üzerindeki etkileri [The effect of perceived acceptance-rejection in childhood on intimate relations in adulthood]. (Unpublished doctoral dissertation). Hacettepe University, Ankara.

Fıșıloğlu, H., \& Demir, A. (2000). Applicability of the dyadic adjustment scale for measurement of marital quality with Turkish couples. European Journal of Psychological Assessment, 16(3), 214-218.

Gökmen, A. (2011). Evli eşlerin birbirine yönelik kontrolcülük ve bağımlılık algılarının evlilik doyumu üzerine etkisi [Dimensions of perception control and dependency effect on marital satisfaction]. (Unpublished master's thesis). Hacettepe University, Ankara.

Graham-Kevan, N. J. A., \& Archer, J. (2008). Does controlling behavior predict physical aggression and violence to partners? Journal of Family Violence, 23, 539-548. https://doi.org/10.1177/ 107780102762478028

Hu, L. T., \& Bentler, P. M. (1999). Cutoff criteria for fit indexes in covariance structure analysis: Conventional criteria versus new alternatives. Structural Equation Modeling, 6(1), 1-55.

Hunt, M. E. (2007). Socioeconomic variables associated with the reports of controlling behaviors in current relationships among abused and non-abused females. (Unpublished master's thesis). University of North Texas, Texas.

IBM Corp. (2012). IBM SPSS statistics for windows, version 21.0. Armonk, NY: IBM Corp.

İnal, G. Ș. (2014). The moderating role of sensory-processing sensitivity in the relationship between spousal caregiving, perceived social support and marital quality. (Unpublished master's thesis). Middle East Technical University, Ankara.

Kamp-Dush, C. M., Taylor, M. G., \& Kroeger, R. A. (2008). Marital happiness and psychological well-being across the life course. Family Relations, 57, 211-226.

Khaleque, A., \& Rohner. (2012). Perceived maternal acceptance, behavioral control, and psychological adjustment of youths: A multiethnic study in the United States. In K. J. Ripoll-Núňez, A. L. Comunian, \& C. M. Brown (Eds.), Expanding horizons: Current research on interpersonal acceptance. Florida: Brown Walker Press.

Khaleque, A., Rohner, R. P., \& Laukkala, H. (2008). Intimate partner acceptance, parental acceptance, behavioral control, and psychological adjustment among Finnish adults in ongoing attachment relationships. Cross-Cultural Research, 42(1), 35-45. https://doi.org/ 10.1177/1069397107309755.

Lawrence, E., Yoon, J., Langer, A., \& Eunyoe, R. (2009). Is psychological aggression as detrimental as physical aggression? The independent effects of psychological aggression on depression and anxiety symptoms. Violence and Victims, 24(1), 20-35.
MacCallum, R. C., Browne, M. W., Sugawara, H., \& M. (1996). Power analysis and determination of sample size for covariance structure modeling. Psychological Methods, 1(2), 130-149.

McDonald, J. E., Olson, J. R., Lanning, A. H., Goddard, H. W., \& Marshall, J. P. (2018). Effects of religiosity, forgiveness, and spousal empathy on marital adjustment. Marriage \& Family Review, 54(4), 393-416.

Öztürk, G. (2017). Evli bireylerin evlilik uyumu ve evlilik doyumu düzeylerinin aşk düzeyi açısından incelenmesi [Evaluation of marital adjustment and marital satisfaction of married individuals in terms of the amount of love]. (Unpublished master's thesis). İstanbul Arel University, İstanbul.

Proulx, C. M., Helms, H. M., \& Buehler, C. (2007). Marital quality and personal well-being: A meta-analysis. Journal of Marriage and Family, 69, 576-593.

Rohner, R. P. (1986). The warmth dimension: Foundations of parental acceptance-rejection theory. Beverly Hills: Sage Publications.

Rohner, R. P. (2001). Handbook for the study of parental acceptance and rejection. Storrs, CT: Rohner Research Publications.

Rohner, R. P. (2004). The parental acceptance-rejection syndrome: Universal correlates of perceived rejection. American Psychologist, $59,827-840$.

Rohner, R. P. (2005). Intimate partner acceptance-rejection/control questionnaire: Test manual. In R. P. Rohner \& A. Khaleque (Eds.), Handbook for the study of parental acceptance and rejection (4th ed., pp. 227-235). Storrs, CT: Rohner Research Publications.

Rohner, R. P. (2008). Introduction: Parental acceptance-rejection theory studies of intimate adult relationships. Cross-Cultural Research, 42(5), 5-12. https://doi.org/10.1177/1069397107309749.

Rohner, R. P. (2016). Introduction to interpersonal acceptance-rejection theory (IPARTheory) and evidence. Online Readings in Psychology and Culture, 6(1), 1-40. https://doi.org/10.9707/2307-0919.1055.

Rohner, R. P., \& Ali, S. (2016). Personality assessment questionnaire (PAQ). In Zeigler-Hill, V. \& Shackelford, T. K. (Eds.), Encyclopedia of personality and individual differences. USA: Springer Reference. https://doi.org/10.1007/978-3-319-28099-8 $55-1$.

Rohner, R. P., \& Khaleque, A. (2008). Relations between perceived partner and parental acceptance, behavioral control, and psychological adjustment among heterosexual adult women in the U.S. In F. Columbus (Ed.), Family relations: Behavioral, psychological, and sociological aspect (pp. 187-197). New York: Noca Science Publishers, Inc.

Sacco, W. P., \& Phares, V. (2001). Partner appraisal and marital satisfaction: The role of self-esteem and depression. Journal of Marriage and Family, 63, 504-513.

Shek, D. T. L. (2001). Marital quality and psychological well-being of married adults in a Chinese context. Journal of Genetic Psychology, 156(1), 45-56.

Spainer, G. B. (1976). Measuring dyadic adjustment: New scales for assessing the quality of marriage and similar dyads. Journal of Marriage and the Family, 38(1), 15-22.

Spainer, G. B., \& Cole, C. L. (1976). Toward clarification and investigation of marital adjustment. International Journal of Sociology of the Family, 6, 121-146.

Stith, S. M., Green, N. M., Smith, D. B., \& Ward, D. B. (2007). Marital satisfaction and marital discord as risk markers for intimate partner violence: A meta-analytic review. Journal of Family Violence, 23, 149-160.

Tabachnick, B. G., \& Fidell, L. S. (2014). Using multivariate statistics (6th ed.). Harlov, Essexx: Pearson.

Thompson, B. (1999). Effects of sample size, estimation methods, and model specification on structural equation modeling fit indexes. Structural Equation Modeling: A Multidisciplinary Journal, 6(1), 56-83. https://doi.org/10.1080/10705519909540119. 
Varan, A. (2003). EKAR kuramı değerlendirme araçlarının Türkiye güvenirlik ve geçerlik çalışması [Reliability and validity of Parental Acceptance and Rejection Theory assessment instruments]. Psychiatry department, Ege University (unpublished study).

Varan, A., Rohner, R. P., \& Eryüksel, G. (2008). Intimate partner acceptance, parental acceptance in childhood, and psychological adjustment among Turkish adults in ongoing attachment relationships. Cross-Cultural Research, 42(1), 46-56. https://doi.org/10.1177/ 1069397107309758.

Villeneuve, L., Dargis, L., Trudel, G., Boyer, R., Préville, M., \& Bégin, J. (2014). Daily hassles, marital functioning and psychological distress among community -dwelling older couples. Revue Européenne de Psychologie Appliquée, 64, 251-258.

Weger, H. (2005). Disconfirming communication and self-verification in marriage: Associations among the demand/withdraw interaction pattern, feeling understood, and marital satisfaction. Journal of Social and Personal Relationships, 22(1), 19-31.

Whisman, M. A., \& Bruce, M. L. (1999). Marital dissatisfaction and incidence of major depressive episode in a community sample. Journal of Abnormal Psychology, 108, 674-678.

Wilcox, W. B., \& Nock, S. L. (2006). What's love got to do with it? Equality, equity, commitment, and women's marital quality. Social Forces, 84(3), 1321-1345.

Yeșiltepe, S. S., \& Çelik, M. (2014). Evaluation of marital adjustment of teachers in terms of psychological well-being and some variables. Elementary Education Online, 13(3), 992-1013.

Publisher's Note Springer Nature remains neutral with regard to jurisdictional claims in published maps and institutional affiliations. 